\title{
Multi-bit MRAM storage cells utilizing serially connected perpendicular magnetic tunnel junctions
}

\author{
Piotr Rzeszut, ${ }^{1, \text { a) }}$ Witold Skowroński, ${ }^{1}$ Sławomir Ziętek, ${ }^{1}$ Jerzy Wrona, ${ }^{2}$ and Tomasz Stobiecki ${ }^{1,3}$ \\ 1) AGH University of Science and Technology, Department of Electronics, \\ Al. Mickiewicza 30, 30-059 Kraków, Poland \\ 2) Singulus technologies, Kahl am Main, 63796, Germany \\ ${ }^{3)}$ AGH University of Science and Technology, Faculty of Physics and Applied Computer Science, \\ Al. Mickiewicza 30, 30-059 Kraków, Poland
}

(Dated: 20 July 2021)

Serial connection of multiple memory cells using perpendicular magnetic tunnel junctions (pMTJ) is proposed as a way to increase magnetic random access memory (MRAM) storage density. Multi-bit storage element is designed using pMTJs fabricated on a single wafer stack, with a serial connections realized using top-to-bottom vias. Tunneling magnetoreistance effect above $130 \%$, current induced magnetization switching in zero external magnetic field and stability diagram analysis of single, two-bit and three-bit cells are presented together with thermal stability. The proposed design is easy to manufacture and can lead to increase capacity of future MRAM devices.

\section{INTRODUCTION}

Spin transfer torque magnetoresistive random access memories (STT-MRAM) have numerous advantages over existing storage technologies, including theoretically unlimited endurance, high read and write speeds and ionizing-cosmicradiation resistance 12 . However, state-of-the-art memories have limited capacity due to the fact, that current density needed to switch a cell (typically made of a single magnetic tunnel junction) requires relatively large transistors 3 . Such an obstacle can be overcome using the architecture that incorporates multibit cell driven by a single transistor.

To date, very few practical implementations of multi-bit MRAM cells have been presented 4[5]. This is mainly due to the fact, that efforts were made to produce a single storage element capable of being stable in more than two states, or to produce multiple storage elements on the top of each other ${ }^{6}$. Both of these approaches are very challenging for the process of manufacture.

In this work an alternative approach is proposed - perpendicular magnetic tunnel junctions (pMTJs) $\sqrt{9}$ are connected electrically in series and a multi-state behaviour is observed, that leads to a multi-bit storage capability. Theoretical explanation as well as experimental results (including working three-bit cell) are presented. In addition, such an approach can be implemented to design and fabricate an artificial synapse for neuromorphic computing scheme $e^{8 / 10}-14$.

\section{PRINCIPLES OF OPERATION}

The discussed pMTJs consist of a top free layer (FL), $\mathrm{MgO}$ tunnel barrier and a bottom reference layer (RL), which is magnetically pinned to the synthetic ferromagnet (SyF) 15 . In the proposed serial connection of pMTJs in a storage cell, the top contact of the first element is connected to the bottom contact of the next element (head-to-tail), as shown in the inset of

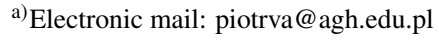

Fig. 1. This results in the charge current flowing through all the cells involved in the same direction. Connections can be made using metallization and vias or any other suitable technique.

The behaviour of the presented arrangement of storage elements can be predicted by analysing characteristics of two pMTJs connected (Fig. 1). If both elements are in the parallel (P) state, the lowest resistance is observed (1). When a positive voltage is applied (which corresponds to the current flow that favours anti-parallel (AP) state), the current increases, until it reaches critical value, which results in the current induced magnetization switching (CIMS) (2). As one of the elements switches to the AP state, with constant voltage applied, the current decreases. This prevents the remaining element of the cell from switching, as the current drops below the critical value. By further increasing the voltage, the critical current is reached again, and the second pMTJ switches to the AP state (3).

By reversing the current polarization, the switching to the $\mathrm{P}$ state is achieved. In this case, as soon as the critical current is reached, one of the elements switches to the P state (4). With constant voltage applied, the current rises above the critical value, causing the other element to switch to $\mathrm{P}$ state.

The above mechanism works also for more than two elements, and similar reasoning can be carried out. For the serial pMTJs connection utilizing the presented mechanism, $N+1$ stable resistance states would be observed for $N$ elements connected, resulting in storage ability of $\log _{2}(N+1)$ bits. This is because there is no possibility to individually determine states of all incorporated storage elements, as ideally they are characterized by the same resistance - only the number of elements in P and AP state may be determined, based on the two-point resistance measurement.

The storage cell capable of storing two bits of data would, therefore, consist of three serially connected storage elements. The predicted resistance vs. voltage characteristics of such a storage cell are presented in Fig. 2 Voltages for writing different states, as well as reading the cell can be defined based on the characteristics obtained for a single pMTJ. Note, that in the proposed cell configuration, writing smaller bit value 


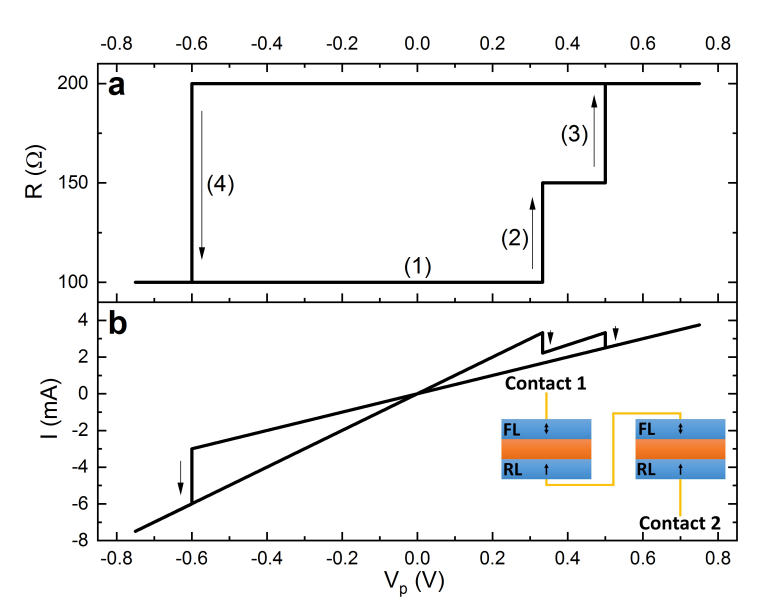

FIG. 1. Theoretically predicted a) resistance and b) current versus voltage applied to a storage cell consisting of two serially connected pMTJs. Inset: Schematic of serial connection of two pMTJs.

(smaller resistance) than the existing state requires clearing the state to "00" (the lowest resistance) and writing a new value.

A simillar solution was suggested by Raymenants et al. in Ref. 5, however, with a different arrangement of subsequent elements, which are connected in opposite directions (headto-head and tail-to-tail). Such a multi-level cell, though, needs application of variable external magnetic field to have an ability to be written with any desired state, what is not a case for our design, where read-write process is much simpler. On the other hand, our solution has by design a limited number of stable states to $N+1$.

Similar mechanism was also suggested by Zhang et. al! 10 , with elements fabricated on top of each other; however, due to experimental difficulties only two working elements connected in series were fabricated.

In our work these drawbacks of other designs are eliminated, and the presented arrangement is ready to be manufactured using unmodified fabrication process.

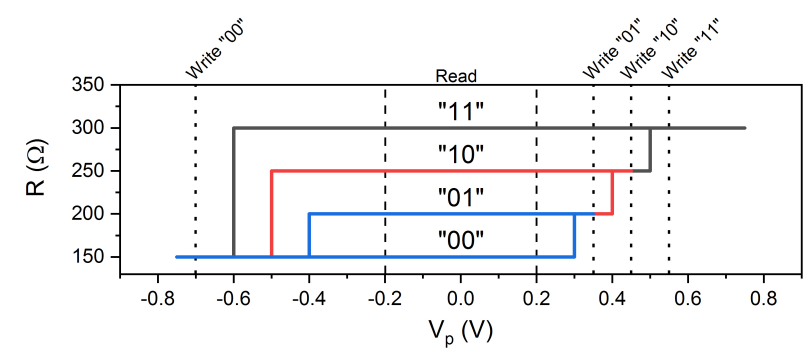

FIG. 2. Theoretically predicted resistance versus voltage applied to the proposed two-bit cell. Possible mapping between resistance and binary value as well as proposed voltages to write and read the cell are presented on the plot. Different colours represent the behaviour of the cell after different writing voltages application.

\section{EXPERIMENT}

Multilayer of the following structure: buffer / $\mathrm{Co}(0.5)-$ $\mathrm{Pt}(0.2)$ based $\mathrm{SyF} / \mathrm{W}(0.25) / \mathrm{CoFeB}(1) / \mathrm{MgO}(0.89) /$ $\mathrm{CoFeB}(1.3) / \mathrm{W}(0.3) / \mathrm{CoFeB}(0.5) / \mathrm{MgO}(0.75) /$ capping layers (thickness in $\mathrm{nm}$ ) patterned into pillars of around $130 \mathrm{~nm}$ diameter were used as pMTJ basic cell. The details of the deposition and fabrication processes are presented in Refs. 1618. Elements were equipped $100 \times 100 \mu \mathrm{m}^{2} \mathrm{Al}(20) / \mathrm{Au}(30)$ contact pads that enable both individual pMTJ characterization as well as measurement of the elements connected in series forming a multi-bit cell. The schematics of the multilayer stack, fabricated pMTJ pillar and a micrograph of a two-bit (three pMTJs in series) cell are presented in Fig. 3 In order to determine an ability of a single element to act as a memory device, two types of characterization were performed: a stability diagram 17 and thermal stability ${ }^{16}$ measurements.

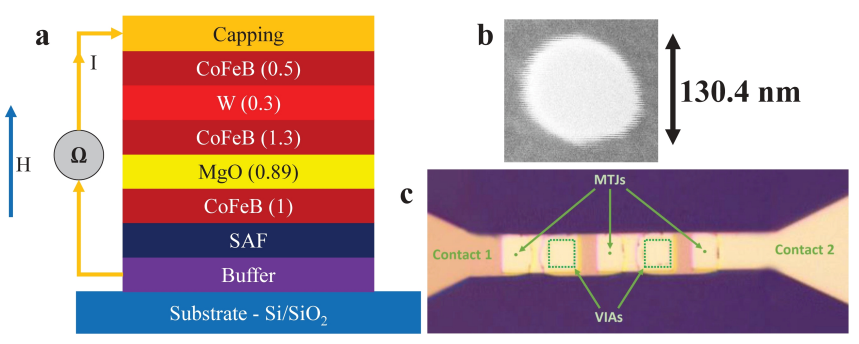

FIG. 3. a) pMTJ layer structure, $S A F$ denotes synthetic antiferromagnet, $H$ - external magnetic field direction, $I$ - current direction, $\Omega$ sourcemeter or cell driving circuit. b) Scanning electron microscope image of a single pMTJ. c) Micrograph of three serially connected pMTJs.

The stability diagram was determined as follows: pMTJ resistance $(R)$ versus voltage pulse amplitude $\left(V_{p}\right)$ measurements were repeated with different external magnetic field $(H)$ applied. Voltage pulse length was set to $10 \mathrm{~ms}$. Each point on the stability diagram corresponds to the transition from the $\mathrm{P}$ to AP state or AP to P state, depending on the initial magnetization configuration. The thermal stability was determined from the $R$ vs. $H$ measurement repeated around hundred times with a fixed sweep rate of around $80 \mathrm{~A} /(\mathrm{ms})$.

\section{RESULTS AND DISCUSSION}

\section{A. Single pMTJ characterization}

An example of the $R\left(V_{p}\right)$ loop and the stability diagram are presented in Fig. 4. The TMR ratio of $135 \%$ and resistance area (RA) product of $21.6 \Omega \mu \mathrm{m}^{2}$ were measured. These values, however, are influenced by series resistance of vias and contacts, which could not be eliminated due to two-wire measurement; in fact, the TMR ratio of the element is higher ${ }^{18}$. In the absence of an external magnetic field, the $\mathrm{P}$ to AP transition occurs for the voltage of around $0.25 \mathrm{~V}$ (corresponding to the critical current density of $2.00 \mathrm{MA} / \mathrm{cm}^{2}$, whereas, the AP 


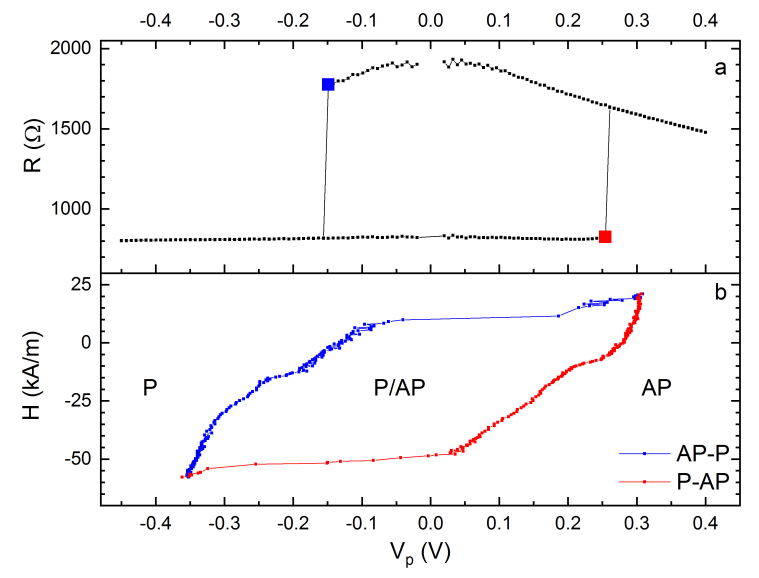

FIG. 4. a) Representative $R$ - $V$ loop of a single pMTJ, with switching voltages from $\mathrm{P}$ to AP (red) and from AP to P (blue) marked using big squares, measured without external magnetic field. b) A stability diagram with marked regions where $\mathrm{P}$, AP or both of the states are stable.

to $\mathrm{P}$ switching is measured for $V_{p}=-0.15 \mathrm{~V}$ (corresponding to $J_{\text {crit }}=-0.54 \mathrm{MA} / \mathrm{cm}^{2}$ ). Multiple $R(H)$ measurements allowed to obtain the switching propability versus $\mathrm{H}$ using the analysis described in Ref. 16 with the following equation

$$
P(\tau)=1-\exp \left[-\frac{\tau}{\tau_{0}} \exp \left\{-\Delta\left(1-\frac{\left|H-H_{s}\right|}{H_{k}^{e f f}}\right)\right\}\right]
$$

In Eq. $11 \tau$ denotes the magnetic field step duration (in this study $\tau=1 \mathrm{~s}$ ), $\tau_{0}$ the inverse of the attempt frequency (in this work assumed to be $1 \mathrm{~ns}$ ), $\Delta$ the thermal stability, $H_{s}$ shift field and $H_{k}^{\text {eff }}$ denotes the effective magnetic anisotropy field.

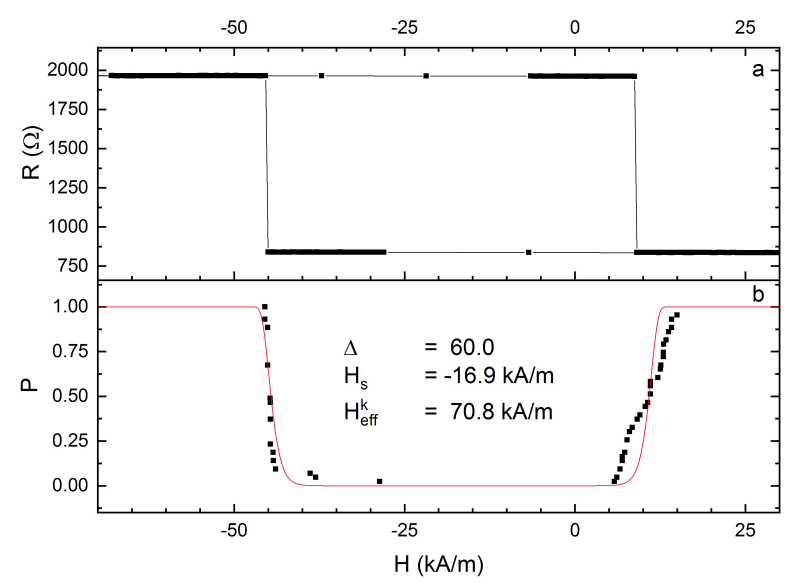

FIG. 5. a) Representative $R-H$ loop. b) Calculated switching probability (black points) and theoretical fit based on Eq. 1

The best fit of Eq. 1 to the experimental switching probability resulted in $\Delta=60$, which, together with a capability of the

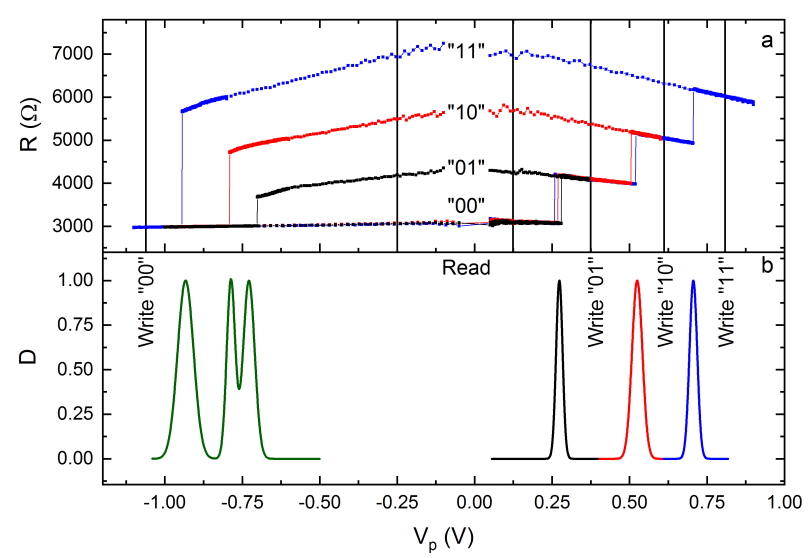

FIG. 6. a) CIMS measurement of two-bit memory cell. The proposed binary coding is also presented. b) Switching voltages distribution, with regions for writing, and safe readout presented.

pMTJ of being stable in both P and AP states in the absence of an external magnetic field proves that the cell is suitable to be used as a memory device.

\section{B. Two-bit storage cell}

Next, we move on to the two-bit cell consisting of three pMTJs connected in series. $R\left(V_{p}\right)$ measurement of such a system is presented in Fig. 6a - initially two-bit cell is in the low resistance state. Application of the positive voltage of around $0.37 \mathrm{~V}$ (corresponding to a single pMTJ switching from $\mathrm{P}$ to AP state) results in the transition to higher resistance state, which is denoted as "01". Further increase of voltage to around $0.67 \mathrm{~V}$ causes a second pMTJ transition to a higher resistance state - thus "10" state is written. Finally, after application of $0.80 \mathrm{~V}$, all three pMTJs are in the AP state, which is denoted as "11" state. Negative voltage of $-1.15 \mathrm{~V}$ switches all pMTJs back to $P$ state. The behaviour described in Sec. II was confirmed - four stable states can be defined and binary numbers can be assigned to them:

- all elements in AP state - 11

- one element in P state and two in AP state - 10

- two elements in AP state and one in P state - 01

- all elements in P state - 00

By repeating $R-V$ measurement of two-bit cell around hundred times and calculating switching voltages distributions, writing voltages of particular states, as well as a region safe for reading the storage cell can be defined (Fig. 60).

The principle of operation, involving the current decreasing below the critical current after one element switching into AP state, was confirmed (Fig. 7). Due to non-ideal manufacturing 


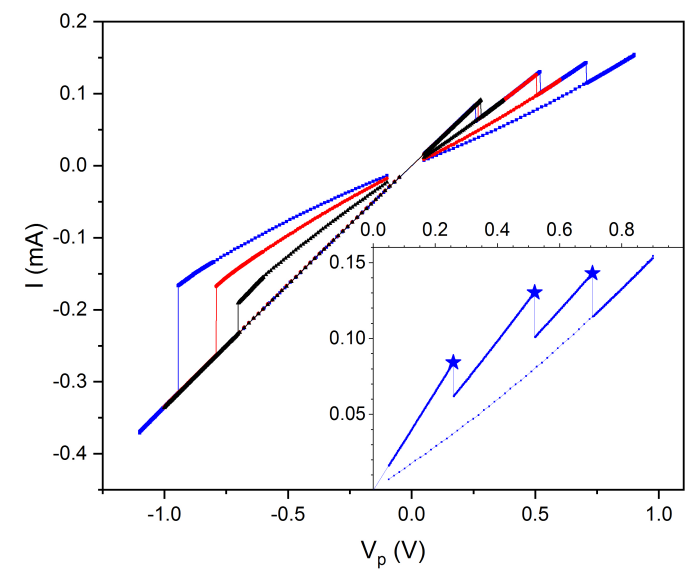

FIG. 7. Current changes during $R-V$ measurement for storage cell constructed of three storage elements. Inset: a close up of current in the CIMS measurement for writing "11" cycle. Critical currents, causing subsequent elements to switch to the AP state are marked with stars.

process, critical currents of all incorporated elements are nonequal, but this has no adverse effect on the process (Fig. 7 inset).

\section{Three-bit storage cell}

Finally, the proof-of-concept of three-bit cell consisting of seven pMTJs connected in series is presented. As predicted, the cell exhibited eight stable states (Fig. 8). Due to nonideal fabrication process it was noted, that regions for writing voltages of some of the states are very narrow because of variation of the switching voltage (related with the switching current distribution). It is believed, that the behaviour is due to very similar critical currents of all incorporated elements. Also switching to "000" state was not ideal in the case, and needs further investigation. Nonetheless, the proposed architecture is valid for a multiple pMTJ that form multi-bit memory cell.

\section{SUMMARY}

In summary, we showed that multi-bit memory cell can be successfully implemented using serially connected pMTJs. State-of-the-art multilayer structure characterized by TMR of $135 \%$ and RA of $21.6 \Omega \mu^{2}$ was used to design two- and three-bit MRAM cells. The developed method of fabrication and driving multi-bit non-volatile storage elements is a significant improvement in MRAM technology, as it allows to store more data using the same area of the memory. This may be achieved by driving a multi-bit storage cell using a single transistor rated for the same current, as a single storage element

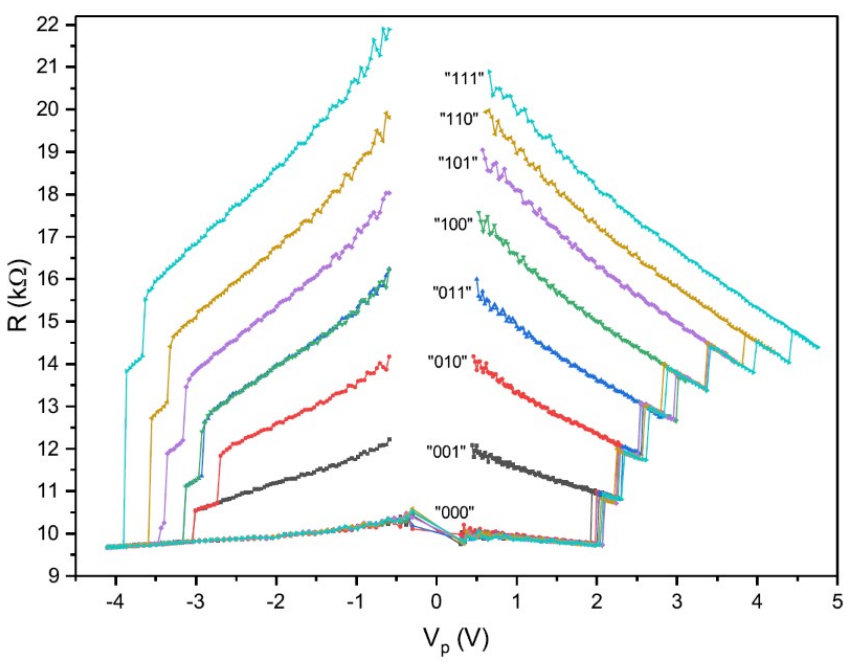

FIG. 8. $R$ - $V$ measurement of three-bit cell consisting of seven pMTJs connected in series. The proposed binary coding is presented.

(the critical current remains the same for any number of serially connected elements). Also, the fabrication process does not require significant changes compared to single storage element fabrication. In addition, the proposed solution maybe utilized in neuromorphic computing scheme as a multi-state non-volatile memory block.

\section{ACKNOWLEDGEMENT}

This work is supported by the Polish Ministry of Science and Higher Education Diamond Grant No. 0048/DIA/2017/46 and the Polish National Centre for Research and Development Grant No. LIDER/467/L-6/14/NCBR/2015.

TS acknowledges the SPINORBITRONICS project through the National Science Centre Poland under grant No. 216/23/B/ST3/01430.

The nano-fabrication process was performed at Academic Centre for Materials and Nanotechnology (ACMiN) of AGH University of Science and Technology.

${ }^{1}$ A. D. Kent and D. C. Worledge, "A new spin on magnetic memories," Nature nanotechnology 10, 187 (2015).

${ }^{2}$ B. Dieny, R. Sousa, J. Herault, C. Papusoi, G. Prenat, U. Ebels, D. Houssameddine, B. Rodmacq, S. Auffret, L. Buda-Prejbeanu, et al., "Spintransfer effect and its use in spintronic components," International Journal of Nanotechnology 7, 591-614 (2010).

${ }^{3}$ T. Kawahara, R. Takemura, K. Miura, J. Hayakawa, S. Ikeda, Y. Lee, R. Sasaki, Y. Goto, K. Ito, T. Meguro, et al., "2Mb spin-transfer torque RAM (SPRAM) with bit-by-bit bidirectional current write and parallelizing-direction current read," in Solid-State Circuits Conference, 2007. ISSCC 2007. Digest of Technical Papers. IEEE International (IEEE, 2007) pp. 480-617.

${ }^{4}$ W.-C. Jeong, B.-I. Lee, and S.-K. Joo, "Three level, six state multilevel magnetoresistive RAM (MRAM)," Journal of applied physics 85, 4782 4784 (1999).

${ }^{5}$ E. Raymenants, A. Vaysset, D. Wan, M. Manfrini, O. Zografos, O. Bultynck, J. Doevenspeck, M. Heyns, I. P. Radu, and T. Devolder, "Chain of magnetic tunnel junctions as a spintronic memristor," Journal of Applied Physics 124, 152116 (2018). 
${ }^{6} \mathrm{~K}$. Ju and O. Allegranza, "Multibit cells schemes for toggle MRAM applications," IEEE transactions on magnetics 42, 2730-2732 (2006).

${ }^{7}$ T. Ishigaki, T. Kawahara, R. Takemura, K. Ono, K. Ito, H. Matsuoka, and H. Ohno, "A multi-level-cell spin-transfer torque memory with seriesstacked magnetotunnel junctions," in 2010 Symposium on VLSI Technology (IEEE, 2010) pp. 47-48.

${ }^{8}$ S. Lequeux, J. Sampaio, V. Cros, K. Yakushiji, A. Fukushima, R. Matsumoto, H. Kubota, S. Yuasa, and J. Grollier, "A magnetic synapse: multilevel spin-torque memristor with perpendicular anisotropy," Scientific reports 6,31510 (2016).

${ }^{9}$ S. Ikeda, K. Miura, H. Yamamoto, K. Mizunuma, H. Gan, M. Endo, S. Kanai, J. Hayakawa, F. Matsukura, and H. Ohno, "A perpendicularanisotropy $\mathrm{CoFeB}-\mathrm{MgO}$ magnetic tunnel junction," Nature materials 9, 721 (2010).

${ }^{10}$ D. Zhang, L. Zeng, K. Cao, M. Wang, S. Peng, Y. Zhang, Y. Zhang, J.-O. Klein, Y. Wang, and W. Zhao, "All spin artificial neural networks based on compound spintronic synapse and neuron," IEEE transactions on biomedical circuits and systems 10, 828-836 (2016).

${ }^{11}$ J. Torrejon, M. Riou, F. A. Araujo, S. Tsunegi, G. Khalsa, D. Querlioz, P. Bortolotti, V. Cros, K. Yakushiji, A. Fukushima, et al., "Neuromorphic computing with nanoscale spintronic oscillators," Nature 547, 428 (2017).

${ }^{12}$ C. Sung, H. Hwang, and I. K. Yoo, "Perspective: A review on memristive hardware for neuromorphic computation," Journal of Applied Physics 124, 151903 (2018).

${ }^{13}$ O. Sulymenko, O. Prokopenko, I. Lisenkov, J. Åkerman, V. Tyberkevych, A. N. Slavin, and R. Khymyn, "Ultra-fast logic devices using artificial "neurons" based on antiferromagnetic pulse generators," Journal of Applied Physics 124, 152115 (2018).

${ }^{14}$ S. Fukami and H. Ohno, "Perspective: Spintronic synapse for artificial neural network," Journal of Applied Physics 124, 151904 (2018).

${ }^{15}$ D. Worledge, G. Hu, D. W. Abraham, J. Sun, P. Trouilloud, J. Nowak, S. Brown, M. Gaidis, E. O'Sullivan, and R. Robertazzi, "Spin torque switching of perpendicular $\mathrm{Ta} / \mathrm{CoFeB} / \mathrm{MgO}$-based magnetic tunnel junctions," Applied Physics Letters 98, 022501 (2011).

${ }^{16}$ H. Sato, M. Yamanouchi, K. Miura, S. Ikeda, R. Koizumi, F. Matsukura, and H. Ohno, "CoFeB thickness dependence of thermal stability factor in $\mathrm{CoFeB} / \mathrm{MgO}$ perpendicular magnetic tunnel junctions," IEEE Magnetics Letters 3, 3000204-3000204 (2012).

${ }^{17}$ W. Skowroński, M. Czapkiewicz, S. Ziętek, J. Chęciński, M. Frankowski, P. Rzeszut, and J. Wrona, "Understanding stability diagram of perpendicular magnetic tunnel junctions," Scientific reports 7, 10172 (2017).

${ }^{18}$ W. Skowroński, S. Łazarski, P. Rzeszut, S. Ziętek, J. Chęciński, and J. Wrona, "Influence of a composite free layer structure on thermal stability of perpendicular magnetic tunnel junction," Journal of Applied Physics 124, 063903 (2018). 